Medicine and sleep

\title{
Disorders of the sleep-wake cycle in adults
}

\author{
Philip M Sedgwick
}

\begin{abstract}
Summary
Adults have an intrinsic body clock which regulates a complex series of rhythms including sleep and wakefulness, fatigue and cognitive ability. This endogenous clock naturally runs more slowly than the solar day and is entrained to a 24-h rhythm primarily by the alternation of light and darkness. Jet lag, shift-work sleep disorder, and some of the chronic insomnias are caused by a temporal discrepancy of the body clock relative to the surrounding environment and social network. The underlying mechanisms and general management are described. Both bright light and melatonin therapy have potential in the management of these disorders. Traditionally, bright light therapy has been used to alleviate the depression associated with seasonal affective disorder. Melatonin has received much illformed publicity, it being claimed that it is a panacea and an 'antiageing' treatment. Both of these treatment approaches are reviewed.
\end{abstract}

Keywords: sleep disorders; circadian clock; bright light therapy; melatonin therapy; jet lag

Departments of Addictive Behaviour, General Psychiatry, and Psychiatry of Disability, St George's Hospital Medical School, Cranmer Terrace, London SW17 ORE, UK PM Sedgwick

\section{The intrinsic body clock}

The concept of an intrinsic body clock is well known. It naturally runs more slowly than the solar day. If isolated from the daylight-dark cycle and all other time cues, sleeping when desired, the adult sleep-wake cycle lengthens to approximately 25 hours $^{1}$ (figure 1). The body clock is termed a circadian clock (from the Latin $c i r c a=a b o u t$, diem =a day). It is synchronised to a $24-\mathrm{h}$ rhythm primarily by the solar day, although social and work cues play a role. These exogenous synchronisers are termed zeitgebers (from the German zeit=time, geber=to give). The body clock not only controls the alternation of sleep and wakefulness but also a complex series of circadian rhythms exhibited in body temperature (figure 2), cardiovascular, endocrine, gastrointestinal, metabolic and respiratory mechanisms.

THE CIRCADIAN RHYTHMICITY OF FATIGUE AND COGNITIVE ABILITY

In addition to the general increase in fatigue that follows sleep deprivation there exists a rhythmic daily cycle governed by the body clock (figure 3A). Fatigue is maximal in the normal time for sleep and minimal in the late afternoon. Cognitive ability shows an inverse relationship. It is minimal in the normal time for sleep and maximal in the late afternoon (figure 3B).

\section{SLEEP DURATION AND PROPENSITY}

Sleep duration and propensity are determined not only by the amount of prior wakefulness but also the body clock. ${ }^{2}$ By convention, the phase of the circadian clock is represented by that of the body temperature rhythm (figure 2). The latter is almost sinusoidal in shape with its nadir between 04.00 and 06.00 hours and zenith from 18.00 to 20.00 hours. Times of sleep-onset are most likely to occur with a major nocturnal and secondary mid-afternoon peak. These 'sleep gates' are phase-locked to the body clock and separated by a 'forbidden zone' for sleep centred at around 21.00 hours. ${ }^{3}$ Sleep-onset normally occurs when body temperature is falling and about six hours before its minimum. Sleep duration will be longest when initiated at this phase. There exists a 'wake-up' zone four to seven hours after the temperature minimum when individuals wake up, regardless of how long they have slept. ${ }^{4}$

\section{Disorders of the sleep-wake cycle}

Disorders of the sleep-wake cycle are characterised by a temporal discrepancy of the circadian clock relative to the surrounding environmental and social zeitgebers. This may arise due to changes in external cues, as in jet lag and shift-work sleep disorder. Equally it can originate because of an abnormality in the timing of the body clock, the result of which is typically chronic insomnia.

JET LAG SYNDROME

The rapid transition across time zones causes a conflict between the body clock and cues that guide it. Upon arrival in the new time zone the environmental and social zeitgebers immediately begin to resynchronise the body clock. The rhythms that the latter controls subsequently follow, although at different rates. The result is jet lag. Symptoms include problems of behaviour and performance (box 1). Their severity is related to the direction of travel and number of time zones crossed.

\section{General management}

Recovery is a natural process. It tends to be slow, particularly in those aged over 50 years. Since the body clock naturally tends to lengthen, adaptation is quicker following westward flights which elongate the traveller's day. It ensues on average 


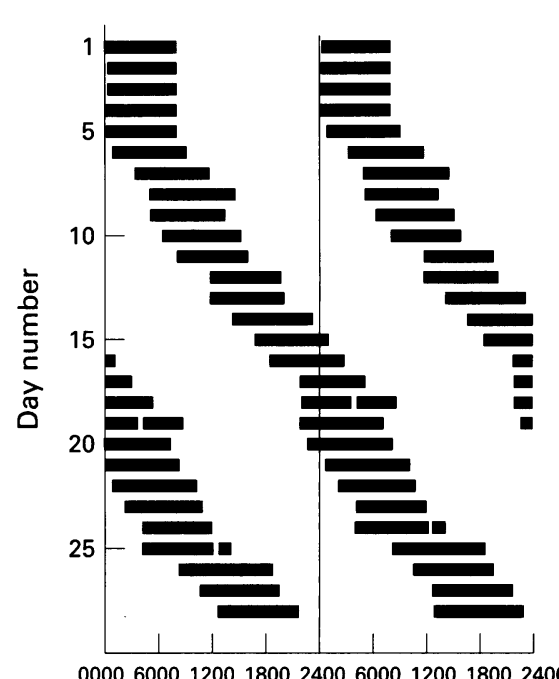

Time of day ( 24 hour clock)

Figure 1 The sleep-wake cycle of a normal adult human in a schedule-free environment (schematic representation). The black bars represent episodes of sleep. The ordinate represents successive 24-h days. The abscissa is 'double-plotted' over $48 \mathrm{~h}$ (top line, day 1 to day 2 ; next line, day 2 to day 3 ; and so on) so as to allow the observation of the uninterrupted procession in the sleep-wake cycle. From day 1 through to 5 , bedtimes and waking times were scheduled on a regular $24-\mathrm{h}$ cycle. Thereafter the subject was allowed to go to bed and arise when they so desired. The sleepwake cycle subsequently 'free-runs' with a period of $25.7 \mathrm{~h}$

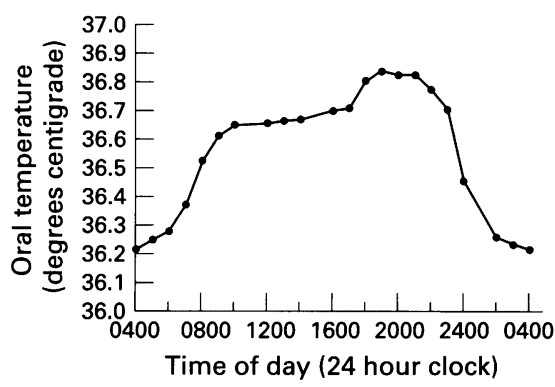

Figure 2 Circadian rhythm of mean oral temperature $\left({ }^{\circ} \mathrm{C}\right)$ for a group of young subjects under constant conditions. By convention, the phase of the circadian clock is represented by that of the body temperature rhythm

\begin{tabular}{|l|}
\hline Symptoms of jet lag \\
\hline - sleep disturbance \\
- daytime fatigue \\
- irregular bowel movements \\
- loss of appetite \\
- headaches \\
cognitive impairment \\
- negative mood states \\
\hline
\end{tabular}

Box 1 at the rate of one and a half time zones per day as opposed to one per day after eastward flights. Adaptation is enhanced by strengthening the cues in the new time zone (box 2). Most importantly, the local hours for sleep and wakefulness should be adopted regardless of fatigue. Alcohol, caffeine, and nicotine all disrupt sleep ${ }^{5}$ and their intake should be limited between four to five hours before bedtime. Correctly timed exposure to natural light during the days immediately after travel helps resynchronise the body clock (table).

SHIFT-WORK SLEEP DISORDER

When work is scheduled nocturnally or on a shift basis, sleep and wakefulness are taken at inappropriate phases of the circadian cycle. The symptoms are similar to jet lag. However, they are exacerbated since the light-dark cycle prevents the body clock from phase-shifting to align with the night-work, day-sleep schedule. ${ }^{6}$

Tolerance to shift work is not simply a biological clock issue centred on the rhythms of sleep propensity, fatigue and performance. A complicated interaction exists with social, domestic and medical factors (box 3). Individuals find it difficult to share domestic responsibilities or pursue hobbies. Marital problems and social isolation are frequently experienced. The long-term impact of shift work upon health is unclear. Sleep disturbances increase after the age of 40 to 50 years. However, the older shift worker tends to have better sleeping and housing conditions, fewer domestic responsibilities and enhanced coping mechanisms.

\section{General management}

There is no best solution or shift system to enhance tolerance. Often the financial gain of companies and individual's livelihoods are at stake making any regime difficult to implement. Education concerning sleep hygiene (box 5) and the influence of circadian factors may be helpful. Alcohol, caffeine and nicotine all disrupt sleep and should be avoided several hours before bedtime. Caffeine taken at the beginning of a shift may improve alertness. Hypnotics should only be used in small doses and then intermittently. Whilst travelling home the wearing of goggles similar to those used by welders has been found to be beneficial. They prevent natural light from impeding the re-alignment of the circadian clock with the work-sleep schedule. ${ }^{7}$

The body clock has a natural tendency to lengthen. Therefore it would suggest that symptoms would be lessened with forward rotating shift systems (ie, evenings, nights, mornings) which elongate the day, as opposed to backward ones (ie, evenings, mornings, nights). A nap during a shift helps stabilise circadian rhythms and assists later performance.

\section{CHRONIC INSOMNIAS}

The delayed and advanced sleep phase syndromes are chronic insomnias associated with abnormal timing of the biological clock. Symptoms of the former include sleep-onset insomnia and/or difficulty in awakening at the desired time. The major sleep episode is at the same time each day but intractably later than socially desired. ${ }^{8}$ Sleep is otherwise normal. Difficulties are often reported as commencing following a period of late night studying or shift work. Although half of such patients display some psychopathology, no particular psychiatric diagnosis is evident. The advanced sleep phase syndrome is characterised by a major sleep period that is persistently earlier than socially desired. ${ }^{9}$ The symptoms are compelling evening sleepiness, with both sleep-onset and final awakening earlier than desired. Sleep is otherwise undisturbed. Too few patients have presented for a psychopathology to be described. Negative personal and social consequences are most likely to eventuate due to the necessity of leaving evening social activities early.

It is assumed that those individuals with the delayed or advanced sleep phase syndrome have a body clock which is delayed or advanced, respectively, in relation to the 24-h clock. So also therefore are their 'sleep gates' and 'forbidden zones'. As with either syndrome there is a weak ability to phase shift the circadian clock in response to environmental and social zeitgebers. Attempts to move the sleep episode to more conventional times usually fail.

The delayed and advanced sleep phase syndromes are apparently rare. Their exact prevalence is not known. However, abnormalities of the circadian time-keeping system are known to be an important component of insomnia. ${ }^{4}$ In particular, initial and early morning insomnia have essentially the same clinical features as the two previously described syndromes, respectively. Primary initial insomniacs have been found to have phase-delayed circadian rhythms and therefore endeavour to fall asleep during their 'forbidden zone' for sleep. ${ }^{10}$ 


\section{Combatting jet lag \\ - adopt the new local hours for sleep and wakefulness regardless of fatigue \\ - avoid naps during the day as they mislead the body clock about night time \\ - rest in a comfortable, quiet and darkened room at bedtime \\ - start the new day with gentle exercises \\ - take part in social functions and adopt regular mealtimes \\ - restrict the intake of alcohol, caffeine and nicotine between four to five hours before bedtime \\ - hypnotics should only be used in small doses and then intermittently}

Box 2

\section{Social, domestic and medical factors that lead to decreased tolerance of shift work}

- over 50 years of age

- second job to alleviate financial problems

- heavy domestic responsibilities

- inadequate housing or sleeping conditions

- history of sleep disorders, psychiatric illness, alcohol or drug abuse

- epilepsy, diabetes, heart disease or gastrointestinal complaints

Box 3
Equally, primary early morning insomniacs have phase-advanced circadian rhythms and attempt to stay asleep during their 'wake-up' zone. ${ }^{11}$

The investigation of disorders of the sleep-wake cycle requires a highly specialised assessment for extended periods of time. Therefore it is likely that a certain proportion of insomniacs are incorrectly diagnosed. It has been estimated that between 5 to $10 \%$ of presenting insomniacs actually have a circadian rhythm disorder.

Table Good and bad local times for exposure to natural daylight in the days immediately following international travel

\begin{tabular}{lcc}
\hline & Bad local times & Good local times \\
\hline Time zones to the west & & \\
$\quad 4$ hours & $01.00-07.00$ & $17.00-23.00$ \\
8 hours & $21.00-03.00$ & $13.00-19.00$ \\
12 hours & $17.00-23.00$ & $09.00-15.00$ \\
Time zones to the east & & \\
$\quad 4$ hours & $01.00-07.00$ & $09.00-15.00$ \\
8 hours & $05.00-08.00$ & $13.00-19.00$ \\
12 hours & $17.00-23.00$ & $09.00-15.00$ \\
\hline
\end{tabular}

\section{Bright light therapy}

The loci of the circadian clock are situated in the suprachiasmatic nuclei of the hypothalamus. Bright light pulses influence the firing rates of specialised neurons located there. Light information is transferred to the suprachiasmatic nuclei via the retinohypothalamic tract, a monosynaptic pathway from the retinal ganglion cells. However, the primary neurotransmitter involved is not known. ${ }^{12}$ Correctly timed exposure to artificial bright white light can advance or delay the circadian clock independently of the sleep-wake cycle. ${ }^{13}$ The phase-relationship between them can therefore be adjusted and subsequently have considerable influence upon the timing, duration and quality of sleep. Morning exposure phaseadvances the circadian clock whilst evening exposure delays it.

Bright light therapy is usually associated with the management of seasonal affective disorder (SAD) (box 5). Most patients find that it alleviates depression and normalises sleep. The phototherapeutic mechanism has not been conclusively demonstrated. It has been hypothesised that circadian rhythms are phase-delayed in winter relative to summer. Therefore morning exposure to bright light would provide corrective advances. However in SAD the effects of light therapy are independent of timing of the circadian phase. ${ }^{14}$ Perhaps evening bright light artificially contracts sleep duration in balance with natural light exposure the following day. Other hypotheses include amplitude enhancement of the circadian clock and simulation of retinal photoreceptor renewal. Equally the antidepressant effect of bright light may merely be a sophisticated placebo or expectation response. ${ }^{15}$

Whilst there have been numerous controlled laboratory investigations of the effects of bright light therapy upon jet lag and shift-work sleep disorder, surprisingly few field studies have been conducted. Those studies which have attempted to alleviate the symptoms of simulated jet lag have produced conflicting results. In particular, unresolved issues exist with regard to timing (pre- or post-flight), duration, and intensity of light exposure. However, post-flight administration at times when exposure to natural daylight is beneficial will no doubt help (table). Appropriately timed bright light at night can alleviate the symptoms of simulated shift work whilst improving mood and later performance. The first published field study of bright light therapy in alleviating the symptoms of shift work detailed its application by NASA. ${ }^{16}$ In order to enhance adaptation to the required night-work schedule of a space shuttle mission, astronauts were exposed to bright light at night during the week before the scheduled night launch. So successful was this implementation that NASA now use light therapy on all space shuttle missions.

Evening light therapy has been successfully used to treat early morning insomniacs. ${ }^{17}$ It delayed their circadian rhythms, including those of melatonin and body temperature, and increased total sleep time. Continued treatment would appear to be necessary. Circadian rhythms tend to phase-advance with ageing leading to an altered phase-relationship with the timing of sleep. ${ }^{18}$ It is thought that this is partly responsible for the sleep maintenance insomnia
Figure 3 Schematic representations of average (A) subjective ratings of fatigue and (B) results of a mental performance task for a group of young subjects whilst awake for three days under constant conditions 


\section{General sleep hygiene instructions}

- sleep in a darkened room

- stay in bed for as long as asleep but no longer

- do not work or watch television in bed; use bed only for sleep and sexual activities

- disruptive environmental factors such as temperature extremes, noise and uncomfortable bed surfaces should be minimised

- take regular exercise, although not immediately before bedtime

- a hot milky drink and/or small snack may aid relaxation and promote more restful sleep

- restrict alcohol, caffeine and tobacco intake, particularly four to five hours prior to bedtime

- avoid the use of hypnotics

Box 4

\section{Symptoms of seasonal affective disorder (SAD)}

SAD has the following symptomatology which regularly occurs in winter and

remits in spring

- sadness

- anxiety

- irritability

- hypersomnia

- increased appetite and carbohydrate craving

- increased weight

- fatigue

- social withdrawal

- decreased libido

- menstrual difficulties characteristic of ageing. The few studies that exist have found that evening light therapy alleviates these symptoms in the healthy elderly. ${ }^{19}$

Bright white light has traditionally been administered using a bank of fluorescent lamps behind a plastic diffusing screen within a metal box. Those light boxes with a large component of spectral emissions in the UV range should be avoided as they can damage the retina. The light box is placed at eye level facing the patient. The individual sits about three feet away whilst watching television or undertaking some similar activity. However, only a small proportion of the transmitted light is actually perceived and this becomes negligible when the distance is increased. The dose obtained is strongly influenced by the user's head position and gaze behaviour. ${ }^{20} \mathrm{~A}$ trade-off relation between intensity and length of exposure is generally assumed. Duration ranges from $30 \mathrm{~min}$ to $4 \mathrm{~h}$ at illuminace levels of 2500 to 10000 lux. (As a frame of reference, domestic light is less than 500 lux whilst sunlight produces 2500 lux just after dawn and 100000 lux at noon.) Head-mounted light visors have recently become available and they eliminate some of the problems experienced with light boxes.

Light boxes and head-mounted visors are commercially available in Britain. The effects of light therapy are not fully known. Reported side-effects include headaches, eyestrain and feeling 'wired' ${ }^{21}$ Furthermore some depressed patients have experienced insomnia and hypermania following treatment. ${ }^{22}$ It is strongly recommended that specialist advice be sought before commencing treatment. Light therapy is open to misuse and abuse, and its incorrect timing can exacerbate the problem.

\section{Melatonin therapy}

Melatonin has been the subject of much ill-formed publicity and speculation in the media world-wide. Assertions that it may be administered as a sleeping pill is far too simplistic. Its long-term effects upon sleep are unknown. Claims for its value as a panacea and 'anti-ageing' treatment have little scientific foundation. The efficacy and safety of melatonin are not known, resulting in it being removed from general sale in the UK. It is available on private prescription or NHS prescription at the discretion of the health authority.

The hormone melatonin is secreted by the pineal gland and regulated by the suprachiasmatic nuclei. Melatonin is normally produced at night and seems to act as a signal of darkness to the body. Bright light suppresses its production although it is not known whether its effects act through this. No function has been definitely associated with melatonin. It is speculated that it is necessary for the regulation of circadian rhythms, reproductive physiology, and the induction of sleep. However, both nocturnal and diurnal animals secrete melatonin at night. Therefore it cannot be directly related to the induction of sleep.

Melatonin is usually administered orally in fast-release dosages of $2-10 \mathrm{mg}$. It has definite phase-setting and entraining effects upon the circadian clock. ${ }^{23}$ When taken in the morning it will result in a phase delay whilst afternoon or early evening administration causes a phase advance. Melatonin acts as a sedative and is therefore able to entrain the sleep-wake rhythm. During the first three or four hours after administration, low doses have rapid, mild and transient effects which result in the lowering of core body temperature. ${ }^{24}$ This in turn reduces arousal and increases sleep propensity.

Melatonin preparations have been used with success in alleviating the subjective and objective symptoms of jet lag. ${ }^{25}$ However, it is of debate whether pre-flight administration is of any benefit. Its timing with regards phase of the circadian clock is crucial as it may lead to major problems in alertness. When taken by shift workers at the desired bedtime, melatonin can reduce sleep-related problems and increase alertness at night. ${ }^{26}$ However, its effects upon work-related performance are unclear.

There is evidence to suggest that some insomnias are a result of a 'melatonin deficiency'. Administration of melatonin at bedtime improved the sleep and daytime alertness in chronic insomniacs. ${ }^{27}$ Biological ageing is known to be associated with disturbed sleep and lowered circulating levels of melatonin. Evening melatonin replacement therapy has been found to lessen sleep disturbances in elderly insomniacs. ${ }^{28}$ Lower levels have also been found in middle-aged primary insomniacs. ${ }^{29}$

\section{Conclusions}

The circadian clock prepares the body for sleep and wakefulness at definite times of the day. If the sleep-wake cycle is shifted causing a temporal discrepancy with the body clock, the results are disrupted sleep and impaired functioning. There are obvious implications. It has been estimated that $27 \%$ of vehicle accidents are 
1 Wever RA. The circadian system of man. Results of experiments under temporal isolation. Berlin experiments under tempo

2 Borbély AA. A two process model of sleep reguBorbely AA. A two process model of sle

3 Lavie P. Ultrashort sleep-waking schedule. III. 'Gates' and 'forbidden zones' for sleep. Electroencephalogr Clin Neurophys 1986;63:414-25.

4 Strogatz SH, Kronauer RE, Czeisler CA. Circadian pacemaker interferes with sleep onset at specific times each day: role in insomnia. $A m \mathcal{F}$ Physiol 1987;253:R172-8.

5 Stradling JR. Recreational drugs and sleep. In: Shapiro CM, ed. $A B C$ of sleep disorders. London: BMJ Publishing Group, 1993; pp 74-6.

6 Monk TH. Shift work. In: Kryger MH, Roth T, Dement WC, eds. Principles and practice of sleep medicine, 2nd edn. Philadelphia: WB Saunders medicine, 2nd edn. Philade

7 Eastman CI, Stewart KT, Mahoney MP, Liu L, Fogg LF. Dark goggles and bright light improve Fogg LF. Dark goggles and bright light improve circadian rhythm adap

Sleep 1994;17:535-43.
8 Weitzman ED, Czeisler CA, Coleman RM, Spielman AJ, Zimmerman JC, Dement WC. Delayed sleep phase syndrome: a chronobiolog cal disorder with sleep-onset insomnia. Arch Gen Psychiatry 1981;38:737-46.

9 Moldofsky H, Musisi S, Phillipson EA. Treatment of a case of advanced sleep phase syndrome by phase advance chronotherapy. Sleep 1986;9:61-5.

10 Morris M, Lack L, Dawson D. Sleep-onset insomniacs have delayed temperature rhythms. Sleep 1990;13:1-14.

11 Lack L, Mercer JD, Wright H. Circadian rhythms of early morning insomniacs. f Sleep Res 1996;5:211-9.

12 Dijk D-J, Boulos Z, Eastman CI, Lewy AJ, Campbell SS, Terman M. Light treatment for

sleep-related. However, they account for $83 \%$ of all fatalities and peak when sleep propensity is greatest. ${ }^{30}$ Those responsible for public health and safety often work night shifts and long hours. They are therefore prone to diminished cognitive ability and/or falling asleep whilst working. Understanding of the mechanisms involved assists in the management of symptoms. Whilst bright light and melatonin therapy are beneficial, more research is needed before their full potential is known.

sleep disorders: consensus report. II Basic properties of circadian physiology and sleep regulation. F Biol Rhythms 1995;10:113-28.

13 Czeisler CA, Allan JS, Strogatz SH, et al. Brigh light resets the human circadian pacemake independent of the timing of the sleep-wake cycle. Science 1986;233:667-71.

14 Wirz-Justice A, Graw P, Krauchi K, et al. Light therapy in seasonal affective disorder is independent of time of day or circadian phase. Arch Gen Psychiatry 1993;50:929-37.

15 Eastman CI, Lahmeyer HW, Watell LG, Good GD, Young MA. A placebo-controlled trial of light treatment for winter depression. $\mathcal{F}$ Affect Disord 1992;26:211-22.

16 Czeisler CA, Chiasera AJ, Duffy JF. Research on sleep, circadian rhythms and ageing: applications to manned spaceflight. Exp Gerontol 1991, 26:217-32.

17 Lack L, Wright $H$. The effect of evening brigh light in delaying the circadian rhythms and lengthening the sleep of early morning awakening insomniacs. Sleep 1993;16:436-43.

18 Weitzman ED, Moline ML, Czeisler CA Zimmerman JC. Chronobiology of ageing: temperature, sleep-wake rhythms and entrainment. Neurobiol Ageing 1982;3:299-309.

19 Campbell SS, Dawson D, Anderson MW. Alleviation of sleep maintenance insomnia with timed exposure to bright light. $\mathcal{F} \mathrm{Am}$ Geriatr Soc 1993;41:829-36.

20 Dawson D, Campbell SS. Bright light treatment: are we keeping our subjects in the dark? Sleep 1990;13:267-71.

21 Levitt AJ, Joffe RT, Moul DE, et al. Side effects of light therapy in seasonal affective disorder. Am $\mathcal{F}$ Psychiatry 1993;150:650-2.
22 Campbell SS, Dijk D-J, Boulos Z, Eastman CI, Lewy AJ, Terman $M$. Light treatment for sleep disorders: consensus report. III Alerting and activating effects. $\mathcal{F}$ Biol Rhythms 1995;10:129 32.

23 Lewy AJ, Ahmed S, Jackson JM, Sack R. Melatonin shifts human circadian rhythms accordin to a phase response curve Chronobiol Int 1992;9:380-92.

24 Deacon S, Arendt J. Melatonin-induced tem perature suppression and its acute phaseshifting effects correlate in a dose-dependen manner in humans. Brain Res 1995;688:77-85.

25 Arendt J, Deacon S, English J, Hampton S, Morgan L. Melatonin and adjustment to phase shift. Work hours, sleepiness and accidents. $f$ Sleep Res 1995;4 (suppl 2):74-9.

26 Folkard S, Arendt J, Clark M. Can melatonin improve shift workers' tolerance of the night shift? Some preliminary findings. Chronobiol Int 1993;10:315-20.

27 MacFarlane JG, Cleghorn JM, Brown GM, Streiner DL. The effects of exogenous melatonin on the total sleep time and daytime alertness of chronic insomniacs: a preliminary study. Biol Psychiatry 1991;30:371-6.

28 Haimov I, Laudon M, Zisapel N, et al. Sleep disorders and melatonin rhythms in elderly people. BMF 1994;309:167.

29 Attenburrow MEJ, Dowling BA, Sharpley AL Cowen PJ. Case-control study of evening melatonin concentration in primary insomnia. $B M \mathcal{F}$ 1996;312:1263-4.

30 Parsons M. Fits and other causes of loss of consciousness while driving. $Q \mathcal{F} \mathrm{Med} 1986 ; 58: 295$ 303. 